\section{Neurodermitis: Dupilumab langfristig überlegen}

Blauvelt $A$ et al. Long-term management of moderate-to-severe atopic dermatitis with dupilumab and concomitant topical corticosteroids (LIBERTY AD CHRONOS): a 1-year, randomised, double-blinded, placebo-controlled, phase-3-trial. Lancet 2017; 389: 2287 - 2303

Hochpotente topische Kortikosteroide und systemische Therapien sind wegen des ungünstigen Nutzen-Risiko-Verhältnisses nicht für eine Dauerbehandlung der Neurodermitis geeignet. Dupilumab reduzierte in Pilotstudien Juckreiz und Hautsymptome. Die FDA ließ den Antikörper deshalb für die therapieresistente Verlaufsform zu. LIBERTY AD CHRONOS war eine internationale Doppelblindstudie, die Nutzen und Sicherheit von Dupilumab nach 16 und 52 Wochen verglich.

Dupilumab bindet an die gemeinsame Untereinheit des Interleukin-4- und Interleukin-13-Rezeptors, hemmt die Signalwege und reduziert damit Typ2/Th2-inflammatorische Antworten. Der monoklonale Antikörper war auch bei Asthma bronchiale und der Polyposis mit chronischer Rhinosinusitis wirksam. An LIBERTY AD CHRONOS nahmen weltweit 161 Zentren teil. In der Phase-3-Parallelgruppenstudie erhielten die Patienten zusätzlich zur topischen Medikation

- Dupilumab 300 mg wöchentlich sc.,

- Dupilumab 300 mg 2-wöchentlich sc. oder

- Placebo wöchentlich sc.

Die vierzehntägige Gruppe bekam in der antikörperfreien Woche Placebo. Als Rescue-Medikation waren topische, systemische und Phototherapy ab der 3. Behand- lungswoche erlaubt. Endpunkte der Studie waren u. a. die Verbesserungen im Investigator's Global Assessment (IGA 0-1/ oder -2), dem Eczema Area and Severity Index (EASI-75) und dem Peak-PruritusScore (NRS-4).

Die Komorbidität mit anderen atopischen Erkrankungen war in allen Gruppen hoch. Wöchentliches Dupilumab erhielten 315, 2-wöchentliches Dupilumab 106 und Placebo 319 Patienten. 52 Wochen Therapie erfolgten bei $86 \%$, 85\% und $67 \%$ der Patienten. 17\%, 16\% und $52 \%$ benötigten die Rescue-Medikation. Mit Dupilumab wurden topische Kortikosteroide an weniger Tagen verwendet. Verglichen mit Placebo hatte Dupilumab nach 16 Wochen die klinische Symptomatik signifikant verbessert. Die Ergebnisse nach 52 Wochen bestätigten die Ergebnisse für wöchentliches und 2-wöchentliches Dupilumab sowie für Placebo:

- IGA $40 \%$ vs. $36 \%$ vs. $13 \%$,

- EASI-75 $64 \%$ vs. $65 \%$ vs. $22 \%$,

- NRS $39 \%$ vs. $51 \%$ vs. $13 \%$.

Der Juckreiz nahm mit Dupilumab bereits nach 2 Wochen deutlich ab. Bei Patienten der Dupilumab-Gruppen waren weniger Körperoberfläche befallen und die Anzahl der Schübe geringer. Auch Allgemeinsymptome wurden günstig beeinflusst. Die Patienten schliefen besser und hatten seltener depressive und Angstsymptome. Dies galt insbesondere in der Gruppe mit einer wöchentlichen Dupilumab-Applikation. Nebenwirkungen des Antikörpers waren Lokalreaktionen und Konjunktivitiden $19 \%$ und $14 \%$, Placebo $8 \%$ ), die überwiegend leicht ausgeprägt waren. Lokalisierte Herpes-simplex-Infektionen kamen in den Dupilumab-Gruppen und Herpes zoster sowie Ekzemata herpeticatum in der PlaceboGruppe häufiger vor.
FAZIT

Die Studie wies einen starken Dupilumab-Effekt nach, der die Relevanz von Interleukin-4 und Interleukin-13 bei der Neurodermitis untermauerte. Die Zytokine dürften laut den Autoren auch bei verwandten atopischen und allergischen Erkrankungen eine Schlüsselrolle einnehmen. Unklar ist die erhöhte Zahl an Konjunktivitiden. Bei Patienten mit anderen atopischen Erkrankungen hatte Dupilumab diese Nebenwirkung nicht. Die Autoren vermuten spezifische NeurodermitisMechanismen, die während der Antikörpertherapie Bindehautentzündungen begünstigten.

Dr. med. Susanne Krome, Melle 